\title{
EFFECT OF FENOFIBRATE ADJUVANT THERAPY ON ENDOTHELIAL DYSFUNCTION AND CARDIOVASCULAR RISK IN EGYPTIAN PATIENTS WITH TYPE 2 DIABETES MELLITUS
}

\author{
SAHAR K HEGAZY ${ }^{1}$, TAMER A ELBEDEWY ${ }^{2}$, HAIDY M SAMI ${ }^{3 *}$ \\ ${ }^{1}$ Department of Clinical Pharmacy, Faculty of Pharmacy, Tanta University, Egypt. ${ }^{2}$ Department of Internal Medicine, Faculty of Medicine, \\ Tanta University, Egypt. ${ }^{3}$ Department of Pharmacy Practice, Faculty of Pharmacy, Delta University for Science and Technology, \\ Gamasa-Mansoura, Egypt. Email: haidysami@outlook.com \\ Received: 28 December 2019, Revised and Accepted: 27 January 2020
}

ABSTRACT

Objective: Endothelial dysfunction is afflicted to the maturation of arteriosclerosis and type 2 diabetes mellitus (T2DM) vascular complications. This study designed to evaluate the impact of fenofibrate adjuvant therapy on endothelial dysfunction and cardiovascular risk in patients with T2DM.

Methods: Seventy-five subjects were recruited from the Internal Medicine Department of Tanta University Hospital. The participants were classified into three groups: Group 1 (control group), 25 healthy subjects; Group 2, 25 T2DM patients received glimepiride/metformin (2:500 mg/day); and Group 3, 25 T2DM patients received glimepiride/metformin (2:500 mg/day)+fenofibrate (300 mg/day). Patients were assessed before and $3 \mathrm{months}$ after intervention for the determination of body mass index (BMI), blood pressure, glycemic picture (hemoglobin A1C \% and homeostatic model assessment of insulin resistant), lipid panel, Castelli's risk index (CRI-I and CRI-II), albumin-to-creatinine ratio (ACR), and endothelial dysfunction biomarkers (asymmetric dimethylarginine [ADMA] and Syndecan-1 (SDC-1)]. Data were statistically analyzed by one-way analysis of variance; $<0.05$ was considered statistically significant.

Results: The addition of fenofibrate to oral hypoglycemic drugs (Group 3) provoked a significant decrease in all measured parameters when compared with Group 2. As compared to the control group, Group 3 showed no significant difference in most measured parameters after 3 months of therapy. For T2DM patients of Group 3, both ADMA and SDC-1 showed a significant positive correlation with all measured parameters except for high-density lipoprotein cholesterol which exhibited significant negative correlation after 3 months of therapy $(\mathrm{p}<0.05)$.

Conclusion: The addition of fenofibrate adjuvant therapy to oral hypoglycemic drugs improved the vascular endothelial dysfunction, CRI-I, CRI-II, glycemic picture, lipid panel, ACR, and blood pressure and reduced the BMI in patients with T2DM.

Keywords: Type 2 diabetes mellitus, Fenofibrate, Endothelial dysfunction, Syndecan-1, Asymmetric dimethylarginine, Castelli's risk index.

(C) 2020 The Authors. Published by Innovare Academic Sciences Pvt Ltd. This is an open access article under the CC BY license (http://creativecommons. org/licenses/by/4. 0/) DOI: http://dx.doi.org/10.22159/ajpcr.2020.v13i3.36733

\section{INTRODUCTION}

Chronic hyperglycemia of diabetes associated with a number of vascular complications [1]. Inflammation is the leading cause in the development of both type 2 diabetes mellitus (T2DM) and insulin resistance (IR). IR in adipose tissue is related to impair repression of lipolysis, which contributes to the hyperlipidemia found in IR state [2]. Dyslipidemia is manifested by raised levels of triglycerides (TGs) carried in very low-density lipoprotein (VLDL) particles, total cholesterol (TC), low high-density lipoprotein cholesterol (HDL-C), and LDL cholesterol (LDL-C) [3]. Other studies demonstrated that in times when the conventional lipid parameters remain apparently normal, lipid ratios such as Castelli's risk index (CRI-I and CRI-II) also known as cardiac risk ratios (CRRs) are the diagnostic alternatives in anticipating the effectiveness of therapy and development of cardiovascular complications [4]. Antecedent studies showed that the excessive uptake of VLDL by macrophage causes foam cell formation and cholesterol accumulation. VLDL clearance occurs primarily through heparan sulfate proteoglycans (HSPGs)-mediated process. Specific HSPGs, like those of the syndecan-1 (SDC-1), contribute to the endocytic clearance of dietary lipids [5].

SDC-1 is also involved in lipoprotein physiology, aiding the clearance of pro-inflammatory chemokine and adhesion molecule. Endothelial cells are covered with SDC-1 and have measurable resistance against compression. Induction of adhesion molecule is accompanied by SDC-1 shedding; however, SDC-1 shedding partially reduces the SDC- 1 thickness and thus found to be insufficient to expose adhesion molecules which are associated with endothelial dysfunction, atherosclerotic lesion, and incident of cardiovascular complications. In addition to its anti-adhesive functions, SDC- 1 also presents neutrophils arrest inducing chemokines [6,7]

Nitric oxide (NO)-mediated vascular reaction is affiliated to endothelial dysfunction and arteriosclerosis maturation [8]. Guanidino-substituted analog of L-arginine blocks the action site of NO-synthase, competitively and inhibits the synthesis of NO. The L-arginine analog is identified as asymmetric dimethylarginine (ADMA) which is equipotent as NO synthase (NOS) inhibitor. Increased levels of ADMA cause impairing in the synthesis of NO, give rise to atherogenesis, endothelial dysfunction, and cardiovascular disease (CVD) [9].

This conundrum could be resolved using drugs that have an impact on endothelial dysfunction, in particular, if it exerts beneficial effect on cardiovascular system [10-12]. Fenofibrate is a peroxisome proliferative activated receptor alpha agonist (PPAR- $\alpha$ ) and has a lot of merits on T2DM vascular complications through its pleiotropic actions $[13,14]$, while the definite mechanism of fenofibrate in endothelial protection is still ambiguous.

The current work was established to determine the fenofibrate safety and efficacy on endothelial dysfunction by the assessment of the levels of SDC-1, ADMA, and cardiovascular risk through the determination of CRI-I and CRI-II in patients with T2DM who never previously administered lipid-lowering medications. 


\section{METHODS}

\section{Subjects}

From January 2017 to May 2018, a total number of 75 subjects who matched according to age, sex, and body mass index (BMI) and fulfilled the criteria of selection were recruited from Internal Medicine Department, Endocrinology Unit at Hospitals of Tanta University, Egypt. National research ethics committee (Tanta University Ethical Committee for Clinical Research) approved the study. This study was carried out in consistence with the ethical standards as laid down in the 1964 Helsinki Declaration and its later amendments or comparable ethical standards. All participants filled out an informed written consent before the beginning of any study procedures. Criteria of patients inclusion: Newly diagnosed T2DM patients controlled on glimepiride/metformin (2:500 mg/day), aged from 18 to 65 years and both gender subjects were included in the study. Criteria of patients exclusion: Patients on lipid-lowering medications, having type 1 diabetes mellitus, fever, autoimmune disease, renal or liver impairment, inflammatory diseases, cancer, acute cardiovascular event within the past 3 months, and uncontrolled endocrine or metabolic disease and patients on drugs that may increase the risk of fenofibrate-induced myopathy (verapamil, diltiazem, amiodarone, cyclosporine, azoles, macrolides, steroids, etc.), all pregnant, breastfeeding women, and women using contraceptives were excluded from the study.

\section{Study design}

The study design was prospective randomized controlled parallel study to evaluate the impact of fenofibrate $300 \mathrm{mg}$ orally/day (Lipanthyl ${ }^{\circledR}$, Fournier Labs France - Minapharm) adjuvant therapy to glimepiride/ metformin (2:500 mg orally/day) (Amary ${ }^{\circledR}$ M $2 \mathrm{mg} / 500 \mathrm{mg}$, Sanofi Aventis, Berlin, Germany) in patients with T2DM through the assessment of its impact on endothelial dysfunction biomarkers (ADMA and SDC-1), CRI-I, CRI-II, clinical parameters (BMI and blood pressure), glycemic picture (fasting blood glucose [FBG], fasting insulin, homeostatic model assessment of insulin resistant [HOMA-IR], and hemoglobin A1C [HbA1C \%]), lipid panel, and albumin-to-creatinine ratio (ACR).

The participants were classified into three groups: Group 1 involved 25 healthy subjects (control group) whose attendance is for periodic checkup, Group 2 consisted of 25 T2DM patients received oral glimepiride/metformin (2:500 mg/day) alone, and Group 3 included 25 T2DM patients received oral glimepiride/metformin (2:500 mg/day) plus oral fenofibrate adjuvant therapy $(300 \mathrm{mg} /$ day). The treatment period was 3 months. All patients were followed monthly to check the compliance to the study protocol and report any adverse events.

\section{Demographic and clinical data}

Demographic and clinical data were assessed at baseline. The collected data were age, sex, other health problems, anthropometric measurement (weight, height, and BMI=weight $[\mathrm{kg}] / \mathrm{height}^{2}\left[\mathrm{~m}^{2}\right]$ ), HbA1C \%, and blood pressures (systolic blood pressure [SBP] and diastolic blood pressure [DBP]) (Table 1).

\section{Biochemical assays \\ Samples collection}

Blood samples $(10 \mathrm{ml})$ were collected after $10-12 \mathrm{~h}$ fasting period at baseline for all participants and 3 months after therapy for all patients. HbA1c \% assessment in the whole blood by adding $2 \mathrm{ml}$ of collected blood in tube containing ethylenediaminetetraacetic acid. The remaining $8 \mathrm{ml}$ of blood was centrifuged for $15 \mathrm{~min}$ at $3000 \mathrm{rpm}$ and the serum supernatant was separated immediately. Lipid profile was measured in fresh serum and the remaining serum was divided, coded, and stored at $-80^{\circ} \mathrm{C}$ for biochemical analysis.

First morning, void urine samples were collected at baseline for all participants and 3 months after therapy for all patients.

\section{Blood glucose, insulin, HOMA-IR, and HbA1C}

FBG levels were assayed using the method of glucose oxidase (Spinreact, Spain) [15]. HbA1c \% was measured by ion exchange method (Biosystems, Spain) [16]. Fasting insulin levels were measured using enzyme-linked immunosorbent assay (ELISA) kits (Calbiotech, USA). HOMA-IR index was determined using equation HOMA-IR $=($ FBG $[\mathrm{mg} / \mathrm{dl}] \times$ FBI $[\mathrm{mIU} / \mathrm{L}]) / 405[17]$

\section{Assay of SDC-1 and ADMA}

Assessment of the levels of SDC- 1 and ADMA were determined using ELISA kits according to the manufacturers' instructions (CLOUDCLONE CORP, USA).

\section{Lipid panel}

Serum levels of TC and TGs were measured by enzymatic colorimetric method [18,19] and HDL-C was assessed by precipitation method using commercial kits (BioMed, Germany) [20]. LDL-C was calculated using the Friedewald formula where LDL-C $=(\mathrm{TC}-\mathrm{HDL}-\mathrm{C}-[\mathrm{TGs} / 5])$ provided when the level of TGs is $<400 \mathrm{mg} / \mathrm{dl}$ [21]. The lipid ratios were calculated using the following formulas: $\mathrm{CRI}-1=(\mathrm{TC} / \mathrm{HDL}-\mathrm{C})$ and CRI-II=(LDL-C/HDL). The following are the abnormal values of lipid ratios for cardiovascular risk: CRI-I $>3.5$ in males, $>3.0$ in females and CRI-II >3.3 [22,23].

\section{Microalbuminuria}

Urine samples were analyzed for albumin and creatinine to calculate ACR and determination of microalbuminuria using available immunoturbidometric method assay kits from (Biotecnica S. P. A., Italy) using semiautomatic analyzer.

\section{Statistical analysis}

The collected data were organized and statistically analyzed using the Statistical Package for the Social Sciences (SPSS) software (version 22, SPSS Inc., Chicago, IL, USA). The range, mean, and standard deviation (SD) were calculated for quantitative data. The comparison between two groups and more was done using Chi-square test $\left(\chi^{2}\right)$ and Fisher's exact test for qualitative data. Student's t-test and $\mathrm{Z}$ value of Mann-Whitney U-test were used for comparison between means of

Table 1: Demographic and clinical data of participants at baseline $(n=75)$

\begin{tabular}{|c|c|c|c|c|c|}
\hline \multirow[t]{2}{*}{ Variables } & \multirow{2}{*}{$\begin{array}{l}\text { Group } 1(n=25) \\
n(\%)\end{array}$} & \multirow{2}{*}{$\begin{array}{l}\text { Group } 2(n=25) \\
n(\%)\end{array}$} & \multirow{2}{*}{$\begin{array}{l}\text { Group } 3(n=25) \\
n(\%)\end{array}$} & \multirow[t]{2}{*}{$\chi^{2}$ or $\mathrm{F}$ value } & \multirow[t]{2}{*}{ p-value } \\
\hline & & & & & \\
\hline Male & $9(36.0)$ & $9(36.0)$ & $11(44.0)$ & 0.450 & 0.799 \\
\hline Female & $16(64.0)$ & $16(64.0)$ & $14(56.0)$ & & \\
\hline Hypertension & & $13(52.0)$ & $16(64.0)$ & 0.330 & 0.566 \\
\hline Age (years) & $(43.96 \pm 7.64)$ & $(43.40 \pm 7.52)$ & $(44.92 \pm 7.81)$ & 0.252 & 0.778 \\
\hline Body mass index $\left(\mathrm{kg} / \mathrm{m}^{2}\right)$ & $(30.80 \pm 2.90)$ & $(30.81 \pm 2.87)$ & $(30.90 \pm 3.11)$ & 0.009 & 0.991 \\
\hline Systolic blood pressure (mmHg) & $(126.32 \pm 9.95)$ & $(141.72 \pm 14.25)^{\mathrm{a}}$ & $(146.24 \pm 14.48)^{\mathrm{a}}$ & 15.983 & $0.0001^{* *}$ \\
\hline Diastolic blood pressure (mmHg) & $(75.40 \pm 4.77)$ & $(92.88 \pm 10.78)^{a}$ & $(90.12 \pm 8.13)^{a}$ & 32.286 & $0.0001^{* *}$ \\
\hline HbA1C \% & $(5.70 \pm 0.16)$ & $(6.90 \pm 0.54)^{\mathrm{a}}$ & $(6.90 \pm 0.29)^{a}$ & 48.981 & $0.0001^{* *}$ \\
\hline
\end{tabular}

Data are presented as mean \pm SD, $\left(\chi^{2}\right)$ : Chi-square test, $\mathrm{F}$ value of ANOVA test, Group 1: Healthy subjects (control group); Group 2: T2DM patients received glimepiride/

metformin (2:500 mg/day) alone for 3 months; Group 3: T2DM patients received glimepiride/metformin (2:500 mg/day) plus fenofibrate ( $300 \mathrm{mg} / \mathrm{day}$ ) for $3 \mathrm{months}$.

${ }^{a}$ Significant difference compared to control group ( $\left.\mathrm{p}<0.05\right)$, significant $\left({ }^{*} \mathrm{p}<0.05,{ }^{* *} \mathrm{p}<0.01\right)$. SD: Standard deviation, T2DM: Type 2 diabetes mellitus 
two groups of parametric and non-parametric data of independent samples, respectively. For comparison between means of two related groups (at baseline and 3 months after therapy) of parametric and non-parametric data, paired $t$-test and $\mathrm{Z}$ value of Wilcoxon signed-rank test were used, respectively. F value of ANOVA test and Kruskal-Wallis test $\left(\chi^{2}\right)$ was calculated for comparison between more than two means of parametric and non-parametric data, respectively. Pearson's correlation coefficient ( $\mathrm{r}$ ) analysis was applied to evaluate the correlation between variables. Statistical significance was considered at $\mathrm{p}<0.05$.

\section{RESULTS}

Patients characteristics: There were no significant differences in demographic or anthropometric parameters between all groups at baseline (Table 1).

\section{Metabolic parameters and glycemic picture}

A summary of the mean \pm SD values of variables at baseline and 3 months after drug administration in all groups is presented in Table 2 . After 3 months of administration of oral glimepiride/metformin (2:500 $\mathrm{mg} /$ day) plus oral fenofibrate adjuvant therapy ( $300 \mathrm{mg} /$ day) (Group 3), there was a significant decrease in BMI, SBP, DBP, glycemic picture (FBG, HbA1c \%, and HOMA-IR), ACR, and lipid panel (TC, TGs, VLDL, and LDL-C), while there was a significant increase in HDL-C. For Group 2 treated with oral glimepiride/metformin (2:500 mg/day) alone, there were no significant differences in these parameters, while there was a significant increase in SBP and a significant decrease in HDL-C.

The comparison of three groups before and 3 months after therapy revealed that T2DM patients of Group 2 and Group 3 had significant increase in all measured parameters rather than healthy subjects of Group 1 at baseline ( $\mathrm{p}=0.0001)$. After 3 months of therapy, comparing Group 3 with Group 2, there was a significant decrease in BMI ( $p=0.001)$, blood pressure, FBG, HbA1C \%, HOMA-IR, ACR, TC, TG, VLDL, and LDL-C $(p=0.0001)$, whereas HDL-C had been increased $(p=0.0001)$. Comparing Group 3 with Group 1, there were no significant differences in most measured parameters, while there was a significant decrease in BMI and HDL-C and an increase in LDL-C, FBG, and DBP. Comparing Group 2 with Group 1, there was a significant increase in SBP, DBP, FBG, HbA1C \%, HOMA-IR, TC, TG, VLDL, LDL-C, and ACR ( $\mathrm{p}=0.0001)$ and a decrease in HDL-C $(\mathrm{p}=0.0001)$.

\section{CRR}

After 3 months of administration of oral glimepiride/metformin $(2: 500 \mathrm{mg} /$ day $)$ plus oral fenofibrate adjuvant therapy $(300 \mathrm{mg} /$ day $)$ (Group 3), there was a significant reduction in both CRI-I and CRI-II by $-56.61 \pm 11.55 \%$ and $-66.70 \pm 14.11 \%$, respectively. For Group 2 treated with oral glimepiride/metformin $(2: 500 \mathrm{mg} /$ day $)$ alone, there was a significant increase in both CRI-I and CRI-II by $17.97 \pm 21.99 \%$ and $24.68 \pm 26.74 \%$, respectively.

The comparison of the three groups before and 3 months after therapy revealed that T2DM patients of Group 2 and Group 3 had significant increase in these ratios rather than healthy subjects of Group 1 at baseline ( $\mathrm{p}=0.0001)$. After 3 months of therapy, comparing Group 3 with Group 2, there was a significant decrease in CRI-I and CRI-II $(\mathrm{p}=0.0001)$. Comparing Group 3 with Group 1, there were no significant differences in these ratios. Comparing Group 2 with Group 1, there was a significant increase in CRI-I and CRI-II $(\mathrm{p}=0.0001)$, as shown in Fig. 1 .

\section{Endothelial dysfunction biomarkers}

A summary of the mean \pm SD values of biomarkers at baseline and 3 months after drug administration in all groups is presented in Table 3. After 3 months of administration of oral glimepiride/ metformin $(2: 500 \mathrm{mg} /$ day $)$ plus oral fenofibrate adjuvant therapy (300 mg/day) (Group 3), there were significant decreases in SDC1 and ADMA ( $p=0.0001)$. For Group 2 treated with oral glimepiride/ metformin $(2: 500 \mathrm{mg} /$ day $)$ alone, there were no significant differences in these biomarkers.
The comparison of the three groups before and 3 months after therapy revealed that T2DM patients of Group 2 and Group 3 had significant increase in these biomarkers rather than healthy subjects of Group 1 at baseline ( $\mathrm{p}=0.0001)$. After 3 months, comparing Group 3 with Group 2, there was a significant decrease in both SDC-1 and ADMA ( $p=0.0001)$. Comparing Group 3 with Group 1, there was a significant reduction in SDC- 1 and ADMA by $-43.37 \%$ and $-45.15 \%$, respectively, but still higher than Group 1. Comparing Group 2 with Group 1, there was a significant increase in these biomarkers $(\mathrm{p}=0.0001)$.

\section{Correlation analysis between significantly changed variables}

Pearson's correlation coefficient (r) analysis was applied to measure the correlation between parameters in Group 3 after 3 months of administration of oral fenofibrate adjuvant therapy (300 mg/day) to oral glimepiride/metformin (2:500 $\mathrm{mg} /$ day) revealed that endothelial dysfunction biomarkers (SDC-1 and ADMA) showed a significant positive correlation with CRI-I and CRI-II ( $\mathrm{p}=0.0001)$, as demonstrated in Fig. 2. Furthermore, both SDC-1 and ADMA showed a significant positive correlation with BMI, blood pressure, glycemic picture (FBG, HOMAIR except HbA1C \%, and fasting insulin which showed non-significant positive correlation), ACR, and lipid panel except HDL-C which exhibited significant negative correlation ( $\mathrm{p}=0.0001)$, as presented in Table 4 .

\section{Analysis of side effects and drug interaction}

The current study results revealed that none of patients experienced abdominal disorder, muscle pain, developed gallbladder stone or elevation in liver enzymes, and renal functions as side effects of fenofibrate. Furthermore, none of patients experienced drug interaction of fenofibrate with glimepiride and metformin.

\section{DISCUSSION}

The current study evaluates whether the prescribed PPAR- $\alpha$ agonist (fenofibrate) could have an impact on cardiovascular risk and endothelial dysfunction in patients with T2DM. In this study, the addition of fenofibrate adjuvant therapy to glimepiride/metformin provided highly improvement in the vascular endothelial dysfunction, CRR, glycemic picture, lipid panel, blood pressure, and ACR and exhibited reduction in BMI in patients with T2DM who never previously administered lipid-lowering medications. This improvement was related to non-lipid-lowering effect of fenofibrate on serum endothelial dysfunction biomarkers correlated with its action on free fatty acids, which are endogenous ligands of PPAR- $\alpha$ [24]. The results of in vitro and animal studies that showed a selective PPAR- $\alpha$ agonist exhibited a markedanti-inflammatory effect wereinlinewith thishypothesis $[25,26]$. The dose of fenofibrate has been selected, in this study, which was $300 \mathrm{mg}$, according to the most widely used dose in human [27].

This study revealed that the addition of fenofibrate adjuvant therapy to glimepiride/metformin in T2DM patients for 3 months (Group 3) resulted in significant reduction in BMI when compared with their baseline values and also when compared with Group 2. In accordance with our results, Filippatos et al. concluded the same results of fenofibrate administration in overweight patients [28].

The current work demonstrated a significant reduction in SBP and DBP by the administration of fenofibrate adjuvant therapy to glimepiride/ metformin in T2DM patients for 3 months (Group 3) when compared with Group 2 and their baseline values. In agreement with our results, Koh et al. indicated that addition of fenofibrate therapy resulted in reduction of blood pressure, not only in terms of lipid profile [29].

The first glycemic measure parameters in patients with T2DM are FBG and HbA1C \% $[30,31]$. The present study showed that addition of fenofibrate adjuvant therapy to glimepiride/metformin in T2DM patients for 3 months (Group 3) resulted in significant reduction in these parameters when compared with their baseline values and also when compared with Group 2. Damci et al. supported our study and demonstrated that fenofibrate offers a lot of benefits on glycemic parameters, regardless of lipid level [32]. 


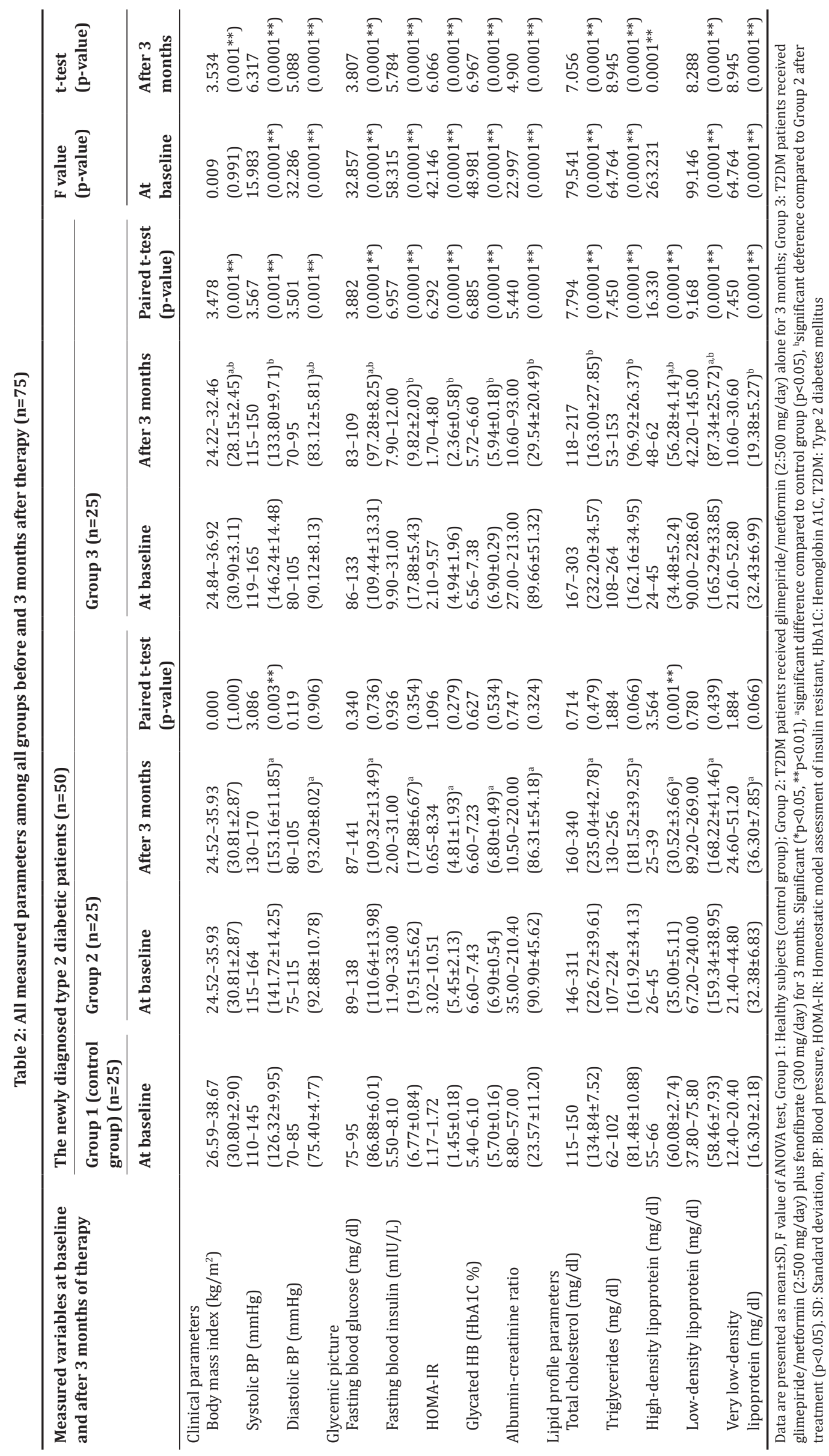




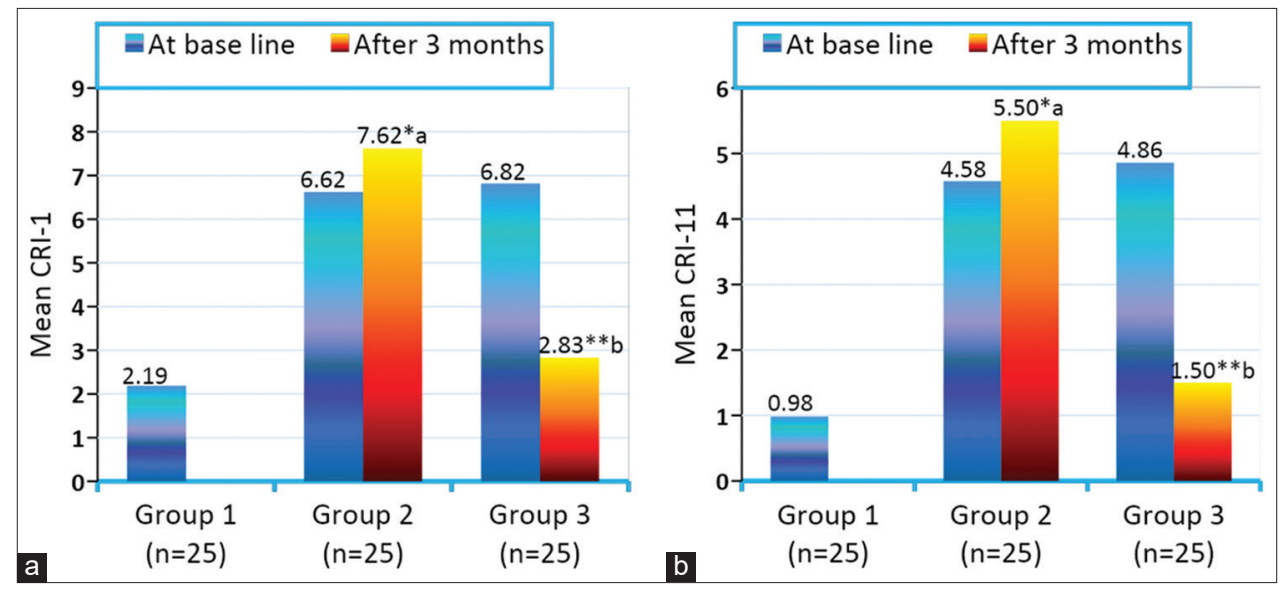

Fig. 1: Mean of Castelli's risk index (CRI-1 and CRI-II). (a) Mean of CRI-I among all groups at baseline and 3 months after therapy (n=75),

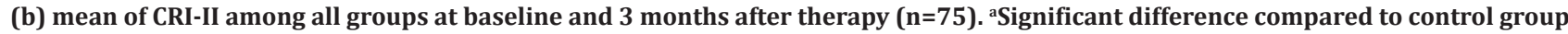
$(p<0.05)$, ${ }^{b}$ significant deference compared to Group 2 after treatment $(p<0.05)$, significant $(* p<0.05, * * p<0.01)$

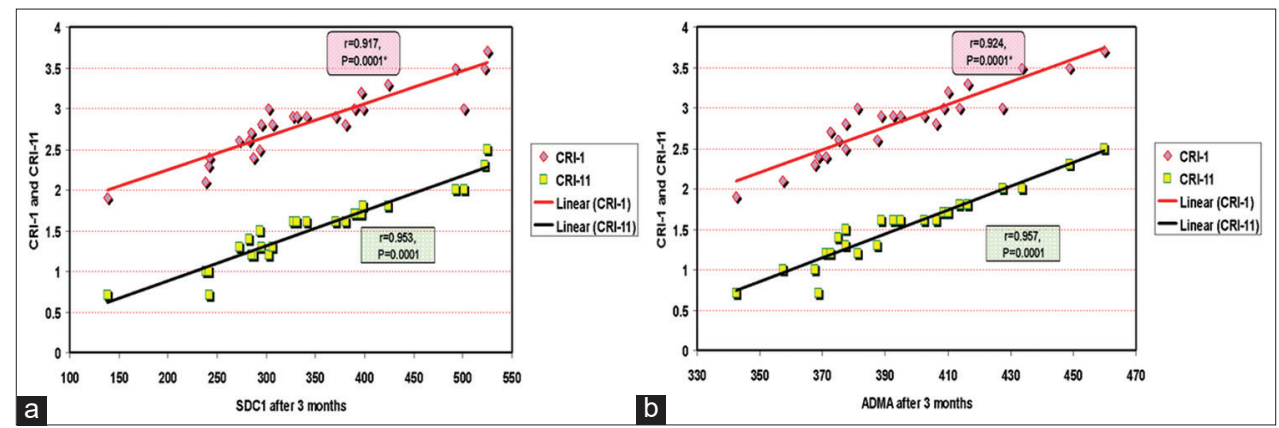

Fig. 2: Pearson's correlation coefficient (r) analysis. (a) Significant positive correlation between Syndecan-1 and Castelli's risk index (CRI-I and CRI-II) after 3 months in Group 3, (b) significant positive correlation between asymmetric dimethylarginine and CRI-I and CRI-II after 3 months in Group 3

Table 3: Endothelial dysfunction biomarkers among all groups at baseline and 3 months after therapy (n=75)

\begin{tabular}{|c|c|c|c|c|c|}
\hline \multirow{3}{*}{$\begin{array}{l}\text { Endothelial } \\
\text { biomarkers at } \\
\text { baseline and } \\
\text { after } 3 \text { months } \\
\text { of therapy }\end{array}$} & \multicolumn{3}{|c|}{ The newly diagnosed type 2 diabetic patients $(n=50)$} & \multirow{3}{*}{$\begin{array}{l}\text { F value } \\
\text { or } t \text {-test }\end{array}$} & \multirow[t]{3}{*}{ p-value } \\
\hline & $\begin{array}{l}\text { Group } 1 \text { (control group) } \\
(n=25)\end{array}$ & Group $2(n=25)$ & Group $3(n=25)$ & & \\
\hline & Range (mean $\pm \mathrm{SD}$ ) & Range (mean $\pm \mathrm{SD}$ ) & Range (mean $\pm \mathrm{SD}$ ) & & \\
\hline \multicolumn{6}{|l|}{ Syndecan-1 (ng/ml) } \\
\hline At baseline & $47.00-190.00(108.38 \pm 30.93)$ & $395.10-755.72(574.65 \pm 103.84)$ & $412.00-790.30(606.92 \pm 111.80)$ & 240.841 & $0.0001^{* *}$ \\
\hline After 3 months & & $368.60-716.10(583.57 \pm 82.53)^{a}$ & $138.77-525.40(343.45 \pm 97.00)^{b}$ & 9.427 & $0.0001^{* *}$ \\
\hline $\begin{array}{l}\text { Paired t-test } \\
\text { (p-value) }\end{array}$ & & $0.336(0.738)$ & $8.900\left(0.0001^{* *}\right)$ & & \\
\hline F value (p-value) & $143.543\left(0.0001^{* *}\right)$ & & & & \\
\hline \multicolumn{6}{|l|}{ ADMA (ng/ml) } \\
\hline At baseline & $77.50-237.50(152.39 \pm 4.46)$ & $614.90-879.90(713.88 \pm 66.21)$ & $632.06-916.00(724.12 \pm 76.43)$ & 643.330 & $0.0001^{* *}$ \\
\hline After 3 months & & $583.90-881.20(744.56 \pm 72.67)^{a}$ & $342.50-460.00(394.40 \pm 28.39)^{b}$ & 22.439 & $0.0001^{* *}$ \\
\hline $\begin{array}{l}\text { Paired t-test } \\
\text { (p-value) }\end{array}$ & & $1.560(0.125)$ & $20.219\left(0.0001^{* *}\right)$ & & \\
\hline F value ( $p$-value) & $466.642\left(0.0001^{* *}\right)$ & & & & \\
\hline
\end{tabular}

Data are presented as mean \pm SD, (F value) of ANOVA test, Group 1: (control group) Healthy subjects; Group 2: T2DM patients received glimepiride/metformin (2:500 mg/day) alone for 3 months; Group 3: T2DM patients received glimepiride/metformin (2:500 mg/day) plus fenofibrate (300 mg/day) for $3 \mathrm{months}$, ${ }^{\mathrm{a}}$ significant difference compared to control group ( $\mathrm{p}<0.05)$, bignificant deference compared to Group 2 after treatment $(\mathrm{p}<0.05)$, significant $\left({ }^{*} \mathrm{p}<0.05,{ }^{* *} \mathrm{p}<0.01\right)$. T2DM: Type 2 diabetes mellitus, ADMA: Asymmetric dimethylarginine

HOMA-IR index is a mathematical calculation from fasting levels of glucose and insulin [33]. This study demonstrated that addition of fenofibrate adjuvant therapy to glimepiride/metformin in T2DM patients for 3 months (Group 3) resulted in significant decrease of fasting insulin level and HOMA-IR index when compared with Group 2 and their baseline values. DeFronzo and Del Prato supported our results and reported that fenofibrate can recover $\beta$-cell function and increase insulin sensitivity in overweight patients [34].

The current results revealed that the level of lipid panel was elevated in patients with T2DM compared with healthy subjects. This study demonstrated that addition of fenofibrate adjuvant therapy to glimepiride/ 
Table 4: Correlation between endothelial dysfunction biomarkers and all measured variables in Group 3 after 3 months of therapy

\begin{tabular}{|c|c|c|c|c|}
\hline \multirow[t]{3}{*}{ All measured variables } & \multicolumn{4}{|c|}{ Endothelial biomarkers among Group 3 after 3 months of therapy $(n=25)$} \\
\hline & \multicolumn{2}{|c|}{ Syndecan-1 } & \multicolumn{2}{|c|}{ Asymmetric dimethylarginine } \\
\hline & r value & p-value & r value & p-value \\
\hline \multicolumn{5}{|l|}{ Clinical parameters } \\
\hline Body mass index & 0.939 & $0.0001^{* *}$ & 0.937 & $0.0001^{* *}$ \\
\hline Systolic blood pressure & 0.861 & $0.0001^{* *}$ & 0.845 & $0.0001^{* *}$ \\
\hline Diastolic blood pressure & 0.619 & $0.001^{* *}$ & 0.649 & $0.001^{* *}$ \\
\hline \multicolumn{5}{|l|}{ Glycemic parameters } \\
\hline Fasting blood glucose & 0.936 & $0.0001^{* *}$ & 0.932 & $0.0001^{* *}$ \\
\hline Fasting insulin & 0.311 & 0.131 & 0.335 & 0.101 \\
\hline HOMA-IR & 0.706 & $0.0001^{* *}$ & 0.640 & $0.001^{* *}$ \\
\hline Glycated $\mathrm{Hb}(\mathrm{HbA} 1 \mathrm{C} \%)$ & 0.095 & 0.650 & 0.131 & 0.534 \\
\hline \multicolumn{5}{|l|}{ Lipid profile parameters } \\
\hline Total cholesterol & 0.964 & $0.0001^{* *}$ & 0.967 & $0.0001^{* *}$ \\
\hline Triglycerides & 0.937 & $0.0001^{* *}$ & 0.928 & $0.0001^{* *}$ \\
\hline High-density lipoprotein & -0.922 & $0.0001^{* *}$ & -0.891 & $0.0001^{* *}$ \\
\hline Low-density lipoprotein & 0.967 & $0.0001^{* *}$ & & \\
\hline Very low-density lipoprotein & 0.943 & $0.0001^{* *}$ & 0.975 & $0.0001^{* *}$ \\
\hline Albumin-creatinine ratio & 0.894 & $0.0001^{* *}$ & 0.950 & $0.0001^{* *}$ \\
\hline
\end{tabular}

Pearson's correlation coefficient $=(\mathrm{r})$, significant $\left({ }^{*} \mathrm{p}<0.05,{ }^{* *} \mathrm{p}<0.01\right)$, Group 3: T2DM patients received glimepiride/metformin $(2: 500 \mathrm{mg} /$ day) plus fenofibrate

(300 mg/day) for 3 months. T2DM: Type 2 diabetes mellitus, HbA1C: Hemoglobin A1C, HOMA-IR: Homeostatic model assessment of insulin resistant

metformin in T2DM patients for 3 months (Group 3) provoked significant decrease in TGs, TC, VLDL, and LDL-C and an increase in HDL-C when compared with their baseline values and also when compared with Group 2. This result is in agreement with the previous reports [35]

The present work supported that the lipid abnormalities appear to be more pronounced as indicated by raised CRR. These lipid ratios could be used for individuals at high risk of CVD, especially when the absolute values of lipid profiles seem normal. CRI-I and CRI-II have been particularly shown to reflect coronary plaques formation [36]. This study demonstrated a significant reduction in these cardiac ratios by the addition of fenofibrate adjuvant therapy to glimepiride/ metformin in T2DM patients for 3 months (Group 3) when compared with Group 2 and their baseline values. In agreement with these results, studies reiterate that CRI-I and CRI-II represent a clinically convenient predictor and indicator for follow-up monitoring in the treatment of T2DM patients with high risk of CVD [37].

These results showed that the level of SDC-1 was significantly elevated in patients with T2DM compared with healthy subjects. Wang et al. agreed with our results regarding SDC-1 in a study revealed that the level of SDC-1 is high and negative correlated with apolipoprotein A1 in T2DM patients [38]. Recent studies have shown that the level of SDC- 1 is higher in T2DM patients with microalbuminuria than in those without microalbuminuria [39]. The previous studies have shown an association between T2DM, endothelial dysfunction, and microalbuminuria [40]. Others demonstrated that the components of endothelial glycocalyx are shed in response to inflammation. Removing SDC- 1 from the cell coat may affect the permeability of endothelium, leading to the leakage of protein [41]. The present study demonstrated that the addition of fenofibrate adjuvant therapy to glimepiride/metformin in T2DM patients for 3 months (Group 3) provoked significant reduction in SDC1 levels as compared to their baseline values and also when compared with Group 2. The current work is considered to be the lead study to evaluate the efficacy of fenofibrate adjuvant therapy to glimepiride/ metformin on SDC-1, based on the best of our knowledge.

Recently, more attention has been laid to ADMA, an endogenous NOS inhibitor, contributing to endothelial dysfunction. The results of the current study showed that the level of ADMA was significantly elevated in patients with T2DM compared with healthy subjects. In accordance with our results, a study has been documented that the level of ADMA is significantly elevated in individuals with atherosclerosis and T2DM [42]. Other study demonstrated that elevated ADMA level was correlated with major cardiovascular events [43]. This study showed that the addition of fenofibrate adjuvant therapy to glimepiride/metformin in T2DM patients for 3 months (Group 3) exhibited significant reduction in ADMA levels as compared to their baseline values and also when compared with Group 2. The potential effect of fenofibrate on ADMA may be secondary to non-lipid-lowering effects such as a potent pleiotropic action through the activation of PPAR $\alpha$ receptor [44]. Yang et al. have shown that pre-treatment with fenofibrate significantly decreased the level of ADMA in rats [45].

The current study results revealed that none of patients experienced abdominal disorder, muscle pain, developed gallbladder stone or elevation in liver enzymes, and renal functions as side effects of fenofibrate. Davis et al. supported our results and reported that there is no evidence for the initial increase in serum creatinine levels which may be attributed to true renal injury [46]. Furthermore, it reported that fenofibrate may delay albuminuria impairment in patients with T2DM which in agreement with our results [47].

In summary, these results revealed that the pro-inflammatory state and increased levels of endothelial dysfunction biomarkers in patients with T2DM may contribute to the development and progression of T2DMrelated vascular complications this may support the previous finding of antioxidant, anti-inflammatory, and antifibrotic effects of fenofibrate. This study indicated that fenofibrate is a promising approach in prevention and treatment of T2DM-related cardiovascular risk and vascular complication.

\section{CONCLUSION}

In patients with T2DM who never previously administered lipidlowering medications, addition of fenofibrate adjuvant therapy to oral hypoglycemic drugs improved the vascular endothelial dysfunction biomarkers, CRR, glycemic picture, lipid panel, blood pressure, and $\mathrm{ACR}$ and reduced the BMI. Therefore, fenofibrate has a direct protective effect on T2DM-related vascular complications.

\section{Limitations}

Further studies would be required to evaluate long-term efficacy and safety of fenofibrate with long duration of treatment on large number of patients, also other PPAR- $\alpha$ agonists could be examined.

\section{ACKNOWLEDGMENTS}

The authors so grateful to all participants of this study, without them, this work would be never accomplished. 


\section{AUTHORS' CONTRIBUTIONS}

All authors participated evenly in this study and approved the final manuscript.

\section{CONFLICTS OF INTEREST}

All authors declare that there are no conflicts of interest.

\section{FUNDING}

This research was not funded by any organization or company.

\section{REFERENCES}

1. Forbes JM, Cooper ME. Mechanisms of diabetic complications. Physiol Rev 2013;93:137-88.

2. Suganami T, Tanaka M, Ogawa Y. Adipose tissue inflammation and ectopic lipid accumulation. Endocr J 2012;59:849-57.

3. Kumar A, Singh V. Atherogenic dyslipidemia and diabetes mellitus: What's new in the management arena? Vasc Health Risk Manag 2010;6:665-9

4. Bhardwaj S, Bhattacharjee J, Bhatnagar MK, Tyagi S. Atherogenic index of plasma, castelli risk index and atherogenic coefficient- new parameters in assessing cardiovascular risk. Int $\mathrm{J}$ Pharm Bio Sci 2013;3:359-64.

5. Ebara T, Conde K, Kako Y, Liu Y, Xu Y, Ramakrishnan R, et al. Delayed catabolism of apoB-48 lipoproteins due to decreased heparan sulfate proteoglycan production in diabetic mice. J Clin Invest 2000;105:1807-18.

6. Hayashida K, Parks WC, Park PW. Syndecan-1 shedding facilitates the resolution of neutrophilic inflammation by removing sequestered CXC chemokines. Blood 2009;114:3033-43.

7. Hwang SJ, Ballantyne CM, Sharrett AR, Smith LC, Davis CE, Gotto AM Jr., et al. Circulating adhesion molecules VCAM-1, ICAM-1 and SE-selectin in carotid atherosclerosis and incident coronary heart disease cases: The Atherosclerosis Risk In Communities (ARIC) study. Circulation 1997;96:4219-25.

8. Cooke JP, Dzau VJ. Nitric oxide synthase: Role in the genesis of vascular disease. Annu Rev Med 1997;48:489-509.

9. Leone A, Moncada S, Vallance P, Calver A, Collier J. Accumulation of an endogenous inhibitor of nitric oxide synthesis in chronic renal failure. Lancet 1992;8793:572-5.

10. Zambon A, Cusi K. The role of fenofibrate in clinical practice. Diab Vasc Dis Res 2007;4 Suppl 3:S15-20

11. Kalsi A, Singh S, Taneja N, Kukal S, Mani S. Current treatments for Type 2 diabetes, their side effects and possible complementary treatments. Int J Pharm Pharm Sci 2015;3:13-8.

12. Walker AE, Kaplon RE, Lucking SM, Russell-Nowlan MJ, Eckel RH, Seals DR. Fenofibrate improves vascular endothelial function by reducing oxidative stress while increasing endothelial nitric oxide synthase in healthy normolipidemic older adults. Hypertension 2012;60:1517-23.

13. Pruski M, Krysiak R, Okopien B. Pleiotropic action of short-term metformin and fenofibrate treatment, combined with lifestyle intervention, in Type 2 diabetic patients with mixed dyslipidemia. Diabetes Care 2009;32:1421-4.

14. Chen XR, Besson VC, Palmier B, Garcia Y, Plotkine M, MarchandLeroux C. Neurological recovery-promoting, anti-inflammatory, and anti-oxidative effects afforded by fenofibrate, a PPAR alpha agonist, in traumatic brain injury. J Neurotrauma 2007;24:1119-31.

15. Kaplan LA, editor. Glucose. In: Clinical Chemistry. St Louis, Toronto: The CV Mosby Co.; 1984. p. 1032-6.

16. Bissé E, Abraham EC. New less temperature-sensitive microchromatographic method for the separation and quantitation of glycosylated hemoglobins using a non-cyanide buffer system. J Chromatogr 1985;344:81-91.

17. Matthews DR, Hosker JP, Rudenski AS, Naylor BA, Treacher DF, Turner RC. Homeostasis model assessment: Insulin resistance and betacell function from fasting plasma glucose and insulin concentrations in man. Diabetologia 1985;28:412-9.

18. Watson D. A simple method for the determination of serum cholesterol. Clin Chim Acta 1960;5:637-43.

19. Fossati P, Prencipe L. Serum triglycerides determined colorimetrically with an enzyme that produces hydrogen peroxide. Clin Chem 1982;28:2077-80.

20. Warnick GR, Wood PD. National Cholesterol Education Program recommendations for measurement of high-density lipoprotein cholesterol: Executive summary. The National Cholesterol Education Program Working Group on Lipoprotein Measurement. Clin Chem 1995;41:1427-33.

21. Friedewald WT, Levy RI, Fredrickson DS. Estimation of the concentration of low-density lipoprotein cholesterol in plasma, without use of the preparative ultracentrifuge. Clin Chem 1972;18:499-502.

22. Oguejiofor OC, Onwukwe CH, Odenigbo CU. Dyslipidemia in Nigeria: Prevalence and pattern. Ann Afr Med 2012;11:197-202.

23. Ogbera AO, Fasanmade OA, Chinenye S, Akinlade A. Characterization of lipid parameters in diabetes mellitus a Nigerian report. Int Arch Med 2009;2:19.

24. Keating GM, Ormrod D. Micronised fenofibrate: An updated review of its clinical efficacy in the management of dyslipidaemia. Drugs $2002 ; 62: 1909-44$

25. Kleemann R, Verschuren L, de Rooij BJ, Lindeman J, de Maat MM, Szalai AJ, et al. Evidence for anti-inflammatory activity of statins and PPARalpha activators in human C-reactive protein transgenic mice in vivo and in cultured human hepatocytes in vitro. Blood 2004;103:4188-94.

26. Staels B, Koenig W, Habib A, Merval R, Lebret M, Torra IP, et al. Activation of human aortic smooth-muscle cells is inhibited by PPARalpha but not by PPARgamma activators. Nature 1998;393:790-3.

27. Koanantakul B, Jeamanukulkit N, Piamsomboon C, Chawantanpipat C, Khanacharoen I. Efficacy and safety of 12-week treatment with fenofibrate $300 \mathrm{mg}$ in Thai dyslipidemic patients. J Med Assoc Thai 2004;87:1281-5

28. Filippatos TD, Kiortsis DN, Liberopoulos EN, Georgoula M, Mikhailidis DP, Elisaf MS. Effect of orlistat, micronised fenofibrate and their combination on metabolic parameters in overweight and obese patients with the metabolic syndrome: The FenOrli study. Curr Med Res Opin 2005;21:1997-2006.

29. Koh KK, Quon MJ, Han SH, Chung WJ, Ahn JY, Kim JA, et al. Additive beneficial effects of fenofibrate combined with candesartan in the treatment of hypertriglyceridemic hypertensive patients. Diabetes Care 2006;29:195-201

30. American Diabetes Association. Classification and diagnosis of diabetes. Diabetes Care 2017;40 Suppl 1:11-24.

31. Haghighatpanah M, Thunga G, Khare S, Mallayasamy S. Correlation of glycosylated hemoglobin levels with fasting and postprandial glucose in South Indian Type 2 diabetic patients. Int J Pharm Pharm Sci 2016;8:285-8.

32. Damci T, Tatliagac S, Osar Z, Ilkova H. Fenofibrate treatment is associated with better glycemic control and lower serum leptin and insulin levels in Type 2 diabetic patients with hypertriglyceridemia. Eur J Intern Med 2003;14:357-60.

33. Giancaterini A, De Gaetano A, Mingrone G, Gniuli D, Liverani E, Capristo E, et al. Acetyl-L-carnitine infusion increases glucose disposal in Type 2 diabetic patients. Metabolism 2000;49:704-8.

34. DeFronzo RA, Del Prato S. Insulin resistance and diabetes mellitus. J Diabetes Complications 1996;10:243-5.

35. Miyazaki T, Shimada K, Miyauchi K, Kume A, Tanimoto K, Kiyanagi $\mathrm{T}$, et al. Effects of fenofibrate on lipid profiles, cholesterol ester transfer activity, and in-stent intimal hyperplasia in patients after elective coronary stenting. Lipids Health Dis 2010;9:122.

36. Nair D, Carrigan TP, Curtin RJ, Popovic ZB, Kuzmiak S, Schoenhagen P, et al. Association of total cholesterol/high-density lipoprotein cholesterol ratio with proximal coronary atherosclerosis detected by multislice computed tomography. Prev Cardiol 2009;12:19-26.

37. Olamoyegun MA, Oluyombo R, Asaolu SO. Evaluation of dyslipidemia, lipid ratios, and atherogenic index as cardiovascular risk factors among semi-urban dwellers in Nigeria. Ann Afr Med 2016;15:194-9.

38. Wang JB, Zhang YJ, Zhang Y, Guan J, Chen LY, Fu CH, et al. Negative correlation between serum syndecan-1 and apolipoprotein A1 in patients with Type 2 diabetes mellitus. Acta Diabetol 2013;50:111-5.

39. Bangstad HJ, Østerby R, Rudberg S, Hartmann A, Brabrand K, Hanssen KF. Kidney function and glomerulopathy over 8 years in young patients with Type I (insulin-dependent) diabetes mellitus and microalbuminuria. Diabetologia 2002;45:253-61.

40. Ha TS, Duraisamy S, Faulkner JL, Kasinath BS. Regulation of glomerular endothelial cell proteoglycans by glucose. J Korean Med Sci 2004;19:245-52

41. Svennevig K, Kolset SO, Bangstad HJ. Increased syndecan-1 in serum is related to early nephropathy in Type 1 diabetes mellitus patients. Diabetologia 2006;49:2214-6.

42. Fard A, Tuck CH, Donis JA, Sciacca R, Di Tullio MR, Wu HD, et al. Acute elevations of plasma asymmetric dimethylarginine and impaired endothelial function in response to a high-fat meal in patients with Type 2 diabetes. Arterioscler Thromb Vasc Biol 2000;20:2039-44. 
43. Lu TM, Ding YA, Lin SJ, Lee WS, Tai HC. Plasma levels of asymmetrical dimethylarginine and adverse cardiovascular events after percutaneous coronary intervention. Eur Heart J 2003;24:1912-9.

44. Okopień B, Cwalina Ł, Haberka M, Kowalski J, Zieliński M, Szwed Z, et al. Pleiotropic effects of micronized fenofibrate in patients with combined hyperlipidemia. Pol Merkur Lekarski 2002;13:465-9.

45. Yang TL, Chen MF, Luo BL, Yu J, Jiang JL, Li YJ. Effect of fenofibrate on LDL-induced endothelial dysfunction in rats. Naunyn
Schmiedebergs Arch Pharmacol 2004;370:79-83.

46. Davis TM, Ting R, Best JD, Donoghoe MW, Drury PL, Sullivan DR, et al. Effects of fenofibrate on renal function in patients with Type 2 diabetes mellitus: The Fenofibrate Intervention and Event Lowering in Diabetes (FIELD) Study. Diabetologia 2011;54:280-90.

47. Jun M, Zhu B, Tonelli M, Jardine MJ, Patel A, Neal B, et al. Effects of fibrates in kidney disease: A systematic review and meta-analysis. J Am Coll Cardiol 2012;60:2061-71. 